\title{
Triggered massive star formation in the LMC HII complex N44
}

\author{
C.-H. R. Chen ${ }^{1}$, Y.-H. Chu ${ }^{1}$, R. A. Gruendl ${ }^{1}$ and F. Heitsch ${ }^{2}$
}

${ }^{1}$ Dept. of Astronomy, Univ. of Illinois, USA; ${ }^{2}$ Dept. of Astronomy, Univ. of Michigan, USA

\begin{abstract}
We have used Spitzer IRAC and MIPS observations of N44 to identify young stellar objects (YSOs). Sixty YSO candidates with masses $\gtrsim 4 M_{\odot}$ are identified. We have compared the distribution of YSOs with those of the ionized gas, molecular clouds, and HI gas to study the properties of star formation.
\end{abstract}

Keywords. stars: formation, stars: pre-main-sequence, Magellanic Clouds, HII regions

The distribution of YSO candidates relative to the ionized gas, molecular clouds (Fukui et al. 2001), and HI gas (Kim et al. 2003) is shown in the figure below. All YSOs are projected within molecular clouds. The majority are found near the peaks of molecular clouds; those off molecular peaks are in HI peaks. About 3/4 of the YSOs are in the two southern clouds associated with massive stars and ionized gas, and the other 1/4 in the northern cloud without much ionized gas, indicating that these YSOs are the firstgeneration massive stars in that cloud. The star formation history and stellar energy feedback may be responsible for the larger velocity dispersions (Mizuno et al. 2001) in the two southern clouds.
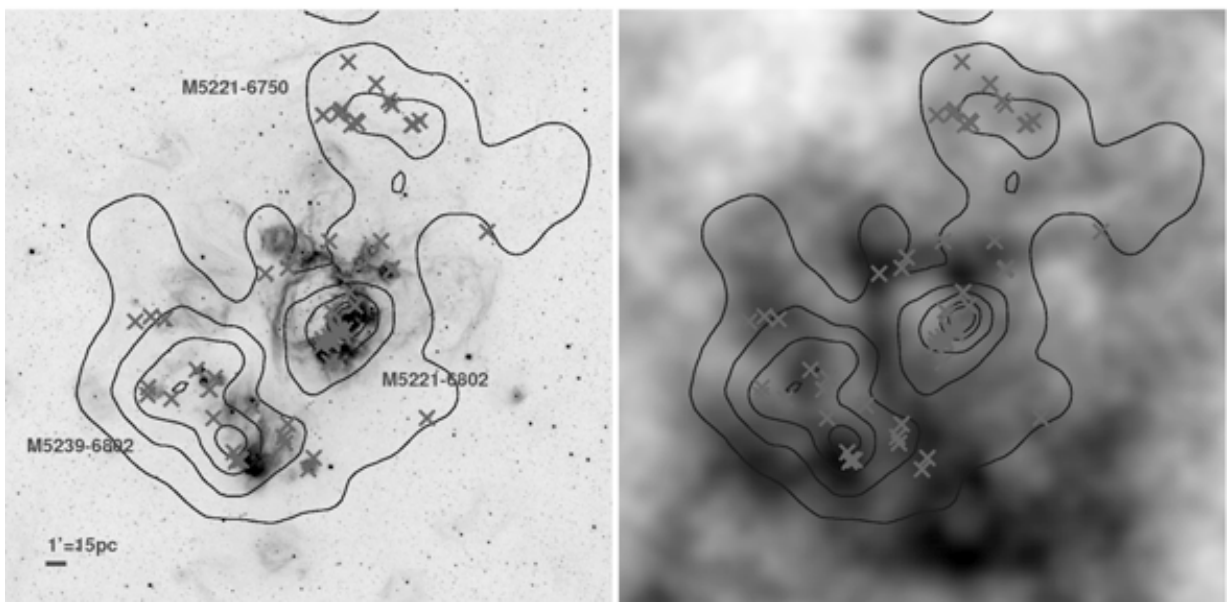

Figure 1. Left: $\mathrm{H} \alpha$ image of N44 marked with YSOs (X), CO contours, and molecular clouds (designation from Mizuno et al. 2001). Right: ATCA+Parkes HI image of N44 (grey scale) marked with YSOs (X) and CO contours.

\section{References}

Fukui, Y., Mizuno, N., Yamaguchi, R., Mizuno, A., \& Onishi, T. 2001, PASJ 53, L41

Kim, S., et al. 2003, ApJS 148, 473

Mizuno, N., et al. 2001, PASJ 53, 971 